\title{
Vitrificación de ovocitos bovinos y su uso en el desarrollo partenogenético de embriones
}

\author{
Vitrification of bovine oocytes and its use in the parthenogenetic \\ development of embryos
}

\author{
J Ruiz ${ }^{\text {a,b, }, c^{*}, \text { JE Correa }}$, M Martínez ${ }^{\mathrm{d}}$ \\ anstituto de Reproducción Animal, Facultad de Ciencias Veterinarias, Universidad Austral de Chile, Valdivia, Chile. \\ bepartamento Académico de Zootecnia, Facultad de Ciencias de Ingeniería, \\ Universidad Nacional de Huancavelica, Huancavelica, Perú. \\ cEstudiante del Programa de Doctorado en Ciencias Veterinarias, Escuela de Graduados, \\ Facultad de Ciencias Veterinarias, Universidad Austral de Chile, Valdivia, Chile. \\ dUnidad de Animales Transgénicos, Genómica Funcional, Centro de Estudios Científicos (CECs), Valdivia, Chile.
}

\begin{abstract}
SUMMARY
The aim of this study was to evaluate vitrification effects on the viability of chemically activated oocytes in order to produce parthenogenetic bovine embryos. Bovine oocytes retrieved from ovaries obtained in a slaughterhouse were matured in vitro for 20-22 hours and then assigned to the following groups: I ( $\mathrm{n}=76)$ : Vitrified/thawed oocytes, II ( $\mathrm{n}=119)$ : exposed oocytes to cryoprotectans without vitrification and III ( $\mathrm{n}=142)$ : control oocytes. Bovine oocytes were vitrified in microdrops on a precooled aluminum foil floating in liquid nitrogen, using an equilibrium solution with $4 \%$ ethylene glycol and a vitrification solution with 35\% ethylene glycol, 5\% polyvinyl-pyrrolidone and 0,4 $\mathrm{M}$ trehalose. The vitrified microdrops were stored in liquid nitrogen and were thawed after 1-3 days of storage. The oocytes of the 3 groups were parthenogenetically activated by 4-min exposure to $5 \mu \mathrm{M}$ Ca ionomycin at room temperature followed through 5 hours incubation in 6-dimethylaminopurine at $38.5^{\circ} \mathrm{C}$ in a $5 \% \mathrm{CO}_{2}$ in humidified atmosphere. Embryos were cultivated on mSOF medium during 8-9 days. The rates of oocytes survival were $55.1 \%$ and $93.7 \%$ to vitrified/thawed (I) and exposed (II) oocytes respectively. The rates of cleavage were $55.3 \%, 72.3 \%$ and $74.6 \%$; and development to blastocysts were $7.1 \%, 17.4 \%$ and $21.7 \%$ in groups I, II and III respectively. These results demonstrate that the oocyte vitrification technique has been set up in our laboratory and parthenogenetic bovine embryos can be produced from such as vitrified/thawed oocytes.
\end{abstract}

Palabras clave: vitrificación, ovocitos, partenogénesis, bovinos.

Key words: vitrification, oocytes, parthenogenesis, bovines.

\section{INTRODUCCIÓN}

La vitrificación es una alternativa a los métodos convencionales de criopreservación. Es un proceso físico de solidificación utilizado para conservar órganos, tejidos y embriones (Cando 2005). La vitrificación es una técnica de congelación ultrarrápida basada en el contacto directo entre la solución de vitrificación que contiene los agentes crioprotectores con las células y el nitrógeno líquido (Rall y Fahy 1985). La definición física de la vitrificación es la solidificación de una solución a baja temperatura sin que ésta llegue a cristalizar debido a un enorme incremento de la viscosidad (Fahy 1986), manteniendo así la distribución molecular e iónica que existía antes de la congelación (Fahy y col 1984). La solución vitrificante utilizada posee crioprotectores en alta concentración que al ser enfriados no cristalizan, sino que se torna viscosa y pasa del estado líquido al sólido no estructurado similar al vidrio, tomando de allí su nombre (Rall y Fahy 1985).

\footnotetext{
Aceptado: 14.10.2009.

* Ciudad Universitaria Paturpampa, Huancavelica, Perú; jaimeruiz@ uach.cl, jarube2801@ gmail.com
}

La estrategia de vitrificación es básicamente diferente a la estrategia de congelación tradicional. Una velocidad lenta de congelación intenta mantener un delicado balance entre varios factores, los cuales pueden resultar en lesiones celulares provocadas por la formación de cristales de hielo, los choques osmóticos, el efecto tóxico de los crioprotectores, la concentración de electrolitos intracelulares, los daños por enfriamiento, las fracturas en la zona pelúcida y las alteraciones de los organelos intracelulares, el citoesqueleto o el contacto entre las células (Massip y col 1995, Dobrinsky 1996, Martino y col 1996). La vitrificación elimina totalmente la formación de cristales de hielo ya que al aumentar la velocidad de congelación disminuyen los daños causados por el enfriamiento pasando rápidamente por el rango de temperatura de mayor peligro entre +15 a $-5^{\circ} \mathrm{C}$ (Dobrinsky 1996, Martino y col 1996, Isachenko y col 1998).

El interés en la criopreservación del ovocito ha aumentado con la creciente importancia en la producción de embriones in vitro, la transferencia nuclear y el almacenamiento de información en los bancos de genes (Dinnyes y col 2000). Por otro lado, el corto tiempo que un ovocito permanece viable y el limitado número de ovocitos que 
pueden colectarse por día hacen que el éxito de la criopreservación de ovocitos mamíferos sea de gran interés para la investigación básica y la aplicación comercial (Albarracín y col 2005). De este modo los ovocitos de algunas especies de mamíferos han sido criopreservados satisfactoriamente a través de procedimientos de congelamiento lento o vitrificación, pero las tasas posteriores de fecundación y desarrollo son mucho más bajas que aquellas obtenidas usando ovocitos frescos (Albarracín y col 2005). Así, Diez y col (2005) obtuvieron 1,6 $\pm 3,2 \%$ blastocistos luego de la fecundación in vitro de ovocitos vitrificados/ descongelados encontrando diferencias significativas con la fecundación in vitro de ovocitos frescos con 36,4 $\pm 3,2 \%$ de blastocistos. Similares resultados reportaron Albarracín y col (2005), con 33,0\% y 2,5\% de blastocistos luego de la fecundación in vitro de ovocitos frescos y vitrificados/ descongelados respectivamente.

La activación es un proceso que se inicia normalmente en el ovocito al momento de ser penetrado por el espermatozoide durante la fecundación (Alberio y Zakhartchenko 2001) elevándose en forma breve y periódica los niveles del calcio intracelular (Tanaka 1997). En la mayoría de mamíferos, el ovocito recién liberado del folículo está detenido en metafase II debido a los altos niveles citoplasmáticos del factor promotor de la maduración (FPM). Una vez que se han fusionado el espermatozoide y el ovocito la liberación de calcio intracelular produce entre otros eventos la inactivación del FPM y el consecuente reinicio y término de la meiosis hasta la extrusión del segundo corpúsculo polar (Williams 2002). La activación química de ovocitos se realiza con el objetivo de imitar en el ovocito los efectos que desencadena la penetración del espermatozoide para estimular el desarrollo de un embrión in vitro (Gonzáles 2007). Por esta razón, esta técnica se utiliza para estimular ovocitos en la producción de embriones partenogenéticos; para activar embriones producidos por inyección intracitoplasmática de espermatozoides (ICSI), por transferencia nuclear (clonación) o transgénesis (Ruiz 2008). Asimismo, es una buena herramienta para evaluar la calidad de los ovocitos madurados in vitro (Gasparrini y col 2004), para evaluar la calidad de ovocitos vitrificados/descongelados (Ruiz y col 2007), para identificar un protocolo óptimo para la activación del ovocito necesaria en la clonación por transferencia nuclear (Gasparrini y col 2004) o para el estudio citogenético de embriones, porque los cromosomas maternos resultantes pueden ser analizados independientemente de los cromosomas paternos (Varga y col 2008).

La vitrificación es una técnica sencilla de ejecutar y que no requiere equipos sofisticados ni caros, por lo que puede montarse fácilmente (Cando 2005). Esta realidad abre la posibilidad de vitrificar ovocitos en lugares apartados y lejanos y de esta manera superar la limitante de la disponibilidad de ovocitos de especies exóticas como camélidos sudamericanos, trasladando estos gametos desde distancias tan lejanas como Putre o Punta Arenas a laboratorios equipados convenientemente en otros lugares de Chile para la producción in vitro de embriones. En nuestro laboratorio se han producido con éxito embriones partenogenéticos con diferentes estímulos químicos y eléctricos (Gatica 2004, Gonzáles 2007). En el presente trabajo se pretende evaluar el efecto de la vitrificación en la viabilidad de ovocitos bovinos activados químicamente para la producción de embriones partenogenéticos.

\section{MATERIAL Y MÉTODOS}

\section{COLECCIÓN Y MADURACIÓN DE COMPLEJO OVOCITO- CUMULUS}

Complejos ovocito-cumulus (COCs) fueron aspirados desde de ovarios de hembras bovinas faenadas en el matadero de Valdivia y fueron madurados in vitro de acuerdo al protocolo descrito por Martínez y col (2007). Brevemente folículos pequeños $(2-7 \mathrm{~mm})$ se aspiraron con agujas $18 \mathrm{G}$ $\mathrm{y}$ jeringas de $10 \mathrm{ml}$ en el laboratorio dentro de las 4 horas siguientes al sacrificio de los animales. El líquido folicular con los COCs recuperados se depositó en tubos cónicos de $50 \mathrm{ml}$ (Falcon, NJ, USA) y fue mantenido a $37^{\circ} \mathrm{C}$ donde se dejó decantar por 20 minutos. El sedimento se aspiró con una pipeta Pasteur y se depositó en placas Petri para ser diluido en medio oviductal sintético modificado, suplementado con 25 mM Hepes (mSOF-Hepes, Takahashi y First 1992) y $1 \mathrm{mg} / \mathrm{ml}$ de albúmina sérica bovina (BSA, Sigma Chemical Co., St. Louis, MO, USA). Los COCs seleccionados bajo una lupa estereoscópica fueron madurados in vitro por 20-22 horas en estufa de cultivo con atmósfera húmeda de $5 \% \mathrm{CO}_{2}$ al aire a $38,5^{\circ} \mathrm{C}$ en gotas de $50 \mu \mathrm{l}(8-12 \mathrm{COCs} /$ gota) de medio de cultivo de tejidos (TCM-199) con $25 \mathrm{mM}$ de Hepes (Sigma) suplementado con piruvato de Na (Sigma) 0,2 mM, sulfato de gentamicina (Sigma) $50 \mu \mathrm{g} / \mathrm{ml}, \mathrm{FSH}$ (Sigma) 0,02 unidades $/ \mathrm{ml}$, estradiol 17- $\beta$ (Sigma) $1 \mu \mathrm{g} / \mathrm{ml}$ y suero fetal bovino (Sigma) al 10\% (v/v). Después de la maduración, para separar los ovocitos de las células del cumulus que los rodean, los COCs fueron agitados mediante vortex por 4 minutos en un tubo Eppendorf de 1,5 ml que contenía solución Dulbecco (DPBS) adicionada de hialuronidasa (Sigma) al 0,1\%. Los ovocitos se lavaron al menos dos veces en mSOF-HEPES y se seleccionaron con la ayuda de un microscopio invertido (Diaphot, Nikon, Tokio, Japan) con aumento de 10x, los que se encontraban en metafase II con un primer corpúsculo polar visible, siendo considerados como ovocitos maduros.

\section{VITRIFICACIÓN DE OVOCITOS}

Se desarrolló un procedimiento de vitrificación en superficie sólida (Dinnyes y col 2000) con algunas modificaciones descritas por Atabay y col (2004). Los ovocitos maduros libres de células del cumulus se suspendieron en un medio de equilibrio (SVI) consistente de $4 \%$ etilenglicol (EG, Sigma) diluido en una solución base (SB) 
compuesta por TCM-199 con 25 mM de Hepes (Sigma) suplementado con $20 \%$ de suero fetal bovino (Sigma) y $50 \mu \mathrm{g} / \mathrm{ml}$ de sulfato de gentamicina (Sigma) por 12-15 minutos sobre una platina temperada a $39^{\circ} \mathrm{C}$. Luego, los ovocitos se colocaron en una solución de vitrificación (SVII) consistente de $35 \%$ EG, $5 \%$ de polivinilpirrolidona (PVP, Sigma) y 0,4 M trehalosa (TRE, Sigma) en SB por 30 segundos. Inmediatamente, microgotas de 3,5 $\mu \mathrm{l}$ de SVII conteniendo 4-6 ovocitos se dejaron caer sobre una superficie de papel de aluminio preenfriado flotando en nitrógeno líquido. Con ayuda de una pinza preenfriada las microgotas vitrificadas fueron transferidas hacia un vial criogénico de plástico de $2 \mathrm{ml}$ preenfriado y fueron almacenadas en nitrógeno líquido, por un tiempo mínimo de 1-3 días hasta su uso.

\section{DESCONGELAMIENTO DE OVOCITOS}

Para descongelar los ovocitos, las microgotas vitrificadas se retiraron del vial criogénico y se expusieron a una solución de $2 \mathrm{ml}$ de TRE $0,3 \mathrm{M}$ en SB por 5 minutos sobre una platina temperada a $39^{\circ} \mathrm{C}$ y luego los ovocitos se transfirieron a SB por un tiempo no menor de 2 minutos. Los ovocitos se lavaron tres veces en mSOF-Hepes y se colocaron en medio de maduración fresco por 30 minutos. De este modo, desde el descongelamiento hasta la activación química transcurrieron aproximadamente 50-60 minutos. Basado en una evaluación morfológica los ovocitos sobrevivientes a la criopreservación se seleccionaron de acuerdo a la integridad de la zona pelúcida y presencia de un ooplasma homogéneo. Los ovocitos dañados por la solución de vitrificación fueron descartados.

\section{ACTIVACIÓN QUÍMICA DE OVOCITOS}

Se formaron tres grupos experimentales de ovocitos expuestos a la activación química: I) ovocitos vitrificados sobrevivientes a la criopreservación, II) ovocitos expuestos a las soluciones vitrificantes (control de toxicidad de los crioprotectantes) y III) grupo control de ovocitos no expuestos a las soluciones vitrificantes. Estos tres grupos experimentales fueron activados de acuerdo al protocolo descrito por Gonzáles (2007). Brevemente, los ovocitos se cultivaron en mSOF-Hepes conteniendo $5 \mu \mathrm{M}$ de ionomicina de $\mathrm{Ca}$ (Sigma) por 4 minutos a $39^{\circ} \mathrm{C}$ e inmediatamente después cultivados en gotas de $100 \mu \mathrm{l}$ de $\mathrm{mSOF}-I V C$ suplementado con $3 \mathrm{mg} / \mathrm{ml}$ de BSA, $2 \mathrm{mM}$ de 6-dimetilaminopurina (6-DMAP, Sigma) y 12,5 $\mu \mathrm{M}$ de citocalasina $\mathrm{B}$ (Sigma) por 5 horas en atmósfera húmeda con $5 \% \mathrm{CO}_{2}$ al aire a $38,5^{\circ} \mathrm{C}$.

\section{CULTIVO IN VITRO DE EMBRIONES}

Concluidos los tratamientos para la activación artificial, los ovocitos se cultivaron de acuerdo a Martínez y col (2007) por 8-9 días en gotas de $40 \mu \mathrm{l}$ de mSOF suplementado con $3 \mathrm{mg} / \mathrm{ml}$ de BSA en estufa de cultivo a $38,5^{\circ} \mathrm{C}$ en atmósfera húmeda con $5 \%$ de $\mathrm{CO}_{2}$ al aire. Se evaluó, con la ayuda de un microscopio invertido (Diaphot, Nikon, Tokyo, Japan) con aumento de 10x, la tasa de segmentación a los dos días y la tasa de blastocistos obtenidos a los ocho días de desarrollo posactivación química de los ovocitos.

\section{ANÁLISIS ESTADÍSTICO}

Los datos obtenidos de segmentación y formación de blastocistos en cada una de las siete réplicas evaluadas fueron expresados en porcentajes. Los resultados fueron analizados utilizando análisis de varianza (ANOVA, una vía) después de la transformación arcsen de los datos. Se utilizó la prueba de Duncan para contrastar la diferencia entre promedios (Valor de significancia: $\mathrm{P}<0,05$ ). Los datos se analizaron utilizando el paquete estadístico SAS versión 8.02 para Windows (1999-2001 por SAS Institute Inc., Cary, NC, USA).

\section{RESULTADOS Y DISCUSIÓN}

Desde el punto de vista morfológico, se logró $55,1 \%$ de supervivencia de ovocitos bovinos a la vitrificación; esta tasa es menor $(\mathrm{P}<0,05)$ a la tasa de supervivencia encontrada con los ovocitos expuestos a las soluciones crioprotectantes y al grupo control no expuesto (cuadro 1). Sin embargo, esta es la primera experiencia realizada en

Cuadro 1. Desarrollo partenogenético de ovocitos bovinos vitrificados, expuestos y no expuestos a una solución vitrificante.

Parthenogenetic development of vitrified, exposed and non-exposed bovine oocytes on the vitrification solution.

\begin{tabular}{lccccc}
\hline Ovocitos & $\mathrm{n}$ & $\begin{array}{c}\text { Sobrevivientes } \\
\text { Post-vitrificación o } \\
\text { post-exposición }(\%)\end{array}$ & $\begin{array}{c}\text { Activados } \\
\text { (Réplicas) } \\
(\%)^{*}\end{array}$ & $\begin{array}{c}\text { Segmentación } \\
2 \text { días } \\
(\%)^{*}\end{array}$ & $\begin{array}{c}\text { blastocistos } \\
8 \text { días } \\
(\%)^{*}\end{array}$ \\
\hline Vitrificados & 138 & $76(55,1 \pm 9,3)^{\mathbf{b}}$ & $76(7)$ & $42(55,3 \pm 5,2)^{\mathbf{b}}$ & $3(7,1 \pm 5,8)^{\mathbf{b}}$ \\
Expuestos & 127 & $119(93,7 \pm 3,7)^{\mathbf{a}}$ & $119(7)$ & $86(72,3 \pm 8,6)^{\mathbf{a}}$ & $15(17,4 \pm 10,4)^{\mathbf{a}, \mathbf{b}}$ \\
No expuestos & 142 & $142(100,0 \pm 0,0)^{\mathbf{a}}$ & $142(7)$ & $106(74,6 \pm 10,5)^{\mathbf{a}}$ & $23(21,7 \pm 9,1)^{\mathbf{a}}$ \\
\hline
\end{tabular}

* Letras diferentes dentro de columnas indican diferencias significativas $(\mathrm{P}<0,05)$. 
nuestro laboratorio, y posiblemente esta tasa incrementará a medida que se tenga un mejor manejo de la técnica, tal como sucedió con Silva y Berland (2004) quienes vitrificaron por primera vez en Chile embriones bovinos con la técnica de Open Pulled Straw (OPS) y obtuvieron tasas de supervivencia posdescongelación menores a las reportadas por otros autores. Asimismo en este trabajo no se alcanzaron las tasas de supervivencia posdescongelación de 88,1\% y $77-86 \%$, reportadas por Atabay y col (2004) y Dinnyes y col (2000) respectivamente; sin embargo, en este estudio se ha demostrado desde el punto de vista fisiológico que los ovocitos están viables posdescongelación expresado en la capacidad de segmentación en respuesta al estímulo de la activación química e incluso se logró la formación de blastocistos (cuadro 1). La diferencia entre nuestros resultados y los reportados por Atabay y col (2004) y Dinnyes y col (2000) se explicaría porque dichos autores evaluaron la supervivencia de los ovocitos inmediatamente después de la descongelación y en este estudio se hizo a los dos días de la activación química, en el momento de la evaluación de la segmentación.

En el cuadro 1 puede observarse también que los crioprotectores usados no fueron tóxicos para los ovocitos bovinos dada la alta tasa de ovocitos morfológicamente normales $(93,7 \%)$ alcanzada luego de la exposición a la solución de vitrificación; esta tasa no fue diferente $(\mathrm{P}>0,05)$ al grupo control de ovocitos no expuestos a la solución de vitrificación. Resultados similares encontraron Dinnyes y col (2000) quienes obtuvieron $94 \%$ de ovocitos sobrevivientes cuando expusieron ovocitos bovinos a la misma solución crioprotectante utilizada en este experimento.

Los ovocitos expuestos y no expuestos a las soluciones crioprotectantes mostraron tasas similares de segmentación $(\mathrm{P}>0,05)$ a los dos días de la activación química (cuadro 1), indicando que los crioprotectores utilizados tampoco afectaron la tasa de segmentación, confirmando los resultados de Dinnyes y col (2000) quienes obtuvieron $69 \%$ y $79 \%$ de segmentación con los ovocitos expuestos y no expuestos a las soluciones crioprotectantes. La vitrificación de ovocitos disminuyó $(\mathrm{P}<0,05)$ la tasa de segmentación $(55,3 \%)$ comparada con los ovocitos expuestos y no expuestos (cuadro 1), lo que refleja algún efecto del procedimiento de vitrificación sobre la segmentación. Al respecto diversos autores explican algunas causas de los efectos de la vitrificación sobre los ovocitos; provocan daño estructural irreversible en la membrana del ovocito (Arav y Zeron 1997), alteran el huso con las consecuentes alteraciones de los cromosomas en los ovocitos en metafase II (Hochi y col 1998, Albarracín y col 2005), alteran la distribución de los gránulos corticales o la distribución de los filamentos de actina (Saunders y Parks 1999, Albarracín y col 2005). Sin embargo, Dinnyes y col (2000) encontraron resultados similares de segmentación $(56,0 \%)$ a los encontrados en este experimento cuando activaron ovocitos bovinos vitrificados/descongelados para la producción de embriones partenogenéticos, lo que indica que el procedimiento de vitrificación utilizado en este experimento fue aplicado correctamente.

Por otro lado, a los ocho días de la activación química se obtuvieron diferencias estadísticas significativas $(\mathrm{P}<0,05)$ en las tasas de blastocistos (cuadro 1). Con el grupo control de ovocitos no expuestos a las soluciones vitrificantes se obtuvo una mayor $(\mathrm{P}<0,05)$ tasa de blastocistos que con los ovocitos vitrificados/descongelados. Las tasas de blastocistos fueron similares $(\mathrm{P}>0,05)$ entre el grupo de ovocitos expuestos a las soluciones crioprotectantes y el grupo de ovocitos vitrificados/descongelados. Al respecto, Dinnyes y col (2000) obtuvieron $11 \%, 25 \%$ y $26 \%$ de blastocistos cuando activaron ovocitos bovinos vitrificados/descongelados, ovocitos expuestos a las soluciones crioprotectantes y ovocitos no expuestos respectivamente, siendo estas tasas de blastocistos relativamente superiores a las reportadas en este experimento; sin embargo esto puede explicarse por las diferentes condiciones de cultivo en ambos experimentos: $5 \%$ de $\mathrm{CO}_{2}$ en la cámara de cultivo utilizada en este experimento frente a una mezcla de gases conformada por $5 \% \mathrm{CO}_{2}, 5 \%$ de $\mathrm{O}_{2}$ y $90 \%$ de $\mathrm{N}_{2}$, utilizada para el cultivo por Dinnyes y col (2000). El mismo grupo de Dinnyes y col (2000) encontró mejores tasas de segmentación y blastocistos cuando utilizó la mezcla de gases conformada por $5 \% \mathrm{CO}_{2}, 5 \%$ de $\mathrm{O}_{2}$ y $90 \%$ de $\mathrm{N}_{2}$ frente al cultivo en una incubadora con $5 \% \mathrm{CO}_{2}$, cuando activaron ovocitos vitrificados/descongelados y ovocitos no vitrificados para la producción de embriones partenogenéticos.

Finalmente, se demuestra que es posible obtener ovocitos bovinos morfológicamente normales luego de la vitrificación/descongelación. La exposición de ovocitos a la solución de vitrificación no mostró reducción en la tasa de segmentación y desarrollo de blastocistos partenogenéticos. Además de la evaluación morfológica, pudo demostrarse que los ovocitos eran fisiológicamente viables posdescongelación, al ser capaces de segmentarse e incluso desarrollarse hasta el estadio de blastocisto. Hasta donde sabemos, estos serían los primeros resultados de vitrificación en superficie sólida de ovocitos bovinos en Chile. Actualmente, está en progreso el estudio de vitrificación de ovocitos de otras especies con esta técnica.

\section{RESUMEN}

El objetivo de este trabajo fue evaluar el efecto de la vitrificación en la viabilidad de ovocitos activados químicamente para la producción de embriones partenogenéticos bovinos. Ovocitos bovinos aspirados de ovarios obtenidos en el matadero fueron madurados in vitro por 20-22 horas y se distribuyeron en los siguientes grupos. I $(n=76)$ : ovocitos vitrificados/descongelados, II ( $\mathrm{n}=119)$ : ovocitos expuestos a crioprotectantes sin vitrificación y III $(n=142)$ : ovocitos control. Los ovocitos fueron vitrificados en microgotas sobre un papel de aluminio preenfriado flotando en nitrógeno líquido, utilizando una solución de equilibrio con $4 \%$ de etilenglicol y una solución de vitrificación con $35 \%$ de etilenglicol 5\% de polivinilpirrolidona y $0,4 \mathrm{M}$ de trehalosa. Las microgotas vitrificadas fueron almacenadas en nitrógeno líquido 
y fueron descongeladas 1-3 días después del almacenamiento. Los tres grupos de ovocitos se activaron partenogenéticamente por exposición de 4 minutos a $5 \mu \mathrm{M}$ de ionomicina de $\mathrm{Ca}$ a temperatura ambiente seguido de una incubación por 5 horas en 6-dimetilaminopurina a $38,5^{\circ} \mathrm{C}$ en una atmósfera húmeda con $5 \% \mathrm{CO}_{2}$. Los embriones se cultivaron en medio mSOF durante 8-9 días. Las tasas de ovocitos sobrevivientes fueron $55,1 \%$ y $93,7 \%$ para ovocitos vitrificados/descongelados (I) y expuestos (II) respectivamente. Las tasas de segmentación de 55,3\%, 72,3\% y 74,6\%, y de desarrollo hasta blastocistos fueron $7,1 \%, 17,4 \%$ y $21,7 \%$ en 1 os grupos I, II y III respectivamente. Estos resultados demuestran que la técnica de vitrificación ha quedado establecida y permite la producción de embriones partenogenéticos bovinos.

\section{AGRADECIMIENTOS}

Parte de este trabajo fue financiado por el proyecto FIA PI-C2004-1-P-047, el proyecto FONDECYT 1050495 y por el Programa de Doctorado en Ciencias Veterinarias de la Universidad Austral de Chile. Jaime Ruiz Béjar agradece a la Cooperación Técnico Belga del Perú por financiar su estadía en Chile.

\section{REFERENCIAS}

Albarracín J, R Morató, C Rojas, T Mogas. 2005. Effects of vitrification in open pulled straws on the cytology of in vitro matured prepubertad and adult bovine oocytes. Theriogenology 63, 890-901.

Alberio R, V Zakhartchenko. 2001. Clonación de animales de interés zootécnico. En: Palma G (ed). Biotecnología de la Reproducción. INTA, Buenos Aires, Argentina, Pp 353-382.

Arav A, Y Zeron. 1997. Vitrification of bovine oocytes using modified minimum drop size technique (MDS) is affected by the composition and concentration of the vitrification solution and by the cooling conditions. Theriogenology 47, 341.

Atabay EC, Y Takahashi, S Katagiri, M Nagano, A Koga, Y Kanai. 2004. Vitrification of bovine oocytes and its application to intergeneric somatic cell nucleus transfer. Theriogenology 61, 15-23.

Cando JF. 2005. Evaluación de la sobrevivencia de embriones de coneja eclosionados, biopsiados y vitrificados por el método open pulled straw modificado. Tesis de Magíster, Facultad de Ciencias Veterinarias, Universidad Austral de Chile, Valdivia, Chile.

Diez C, P Duque, E Gómez, C Hidalgo, C Tamargo, A Rodríguez, L Fernández, S De La Varga, A Fernández, N Facal, M Carbajo. 2005. Bovine oocyte vitrification before or after meiotic arrest: effects on ultrastructure and developmental ability. Theriogenology 64, 317-333.

Dinnyes A, Y Dai, S Jiang, X Yang. 2000. High developmental rates of vitrified bovine oocytes following parthenogenetic activation, in vitro fertilization, and somatic cell nuclear transfer. Biol Reprod 63, 513-518.

Dobrinsky JR. 1996. Cellular approach to cryopreservation of embryos. Theriogenology 45, 17-26.

Fahy G, D MacFarlane, C Angell, H Meryman. 1984. Vitrification as an approach to cryopreservation. Cryobiology 21, 407-426.

Fahy GM 1986. Vitrification: a new approach to organ cryopreservation. Prog Clin Biol Res 224, 305-335.

Gasparrini B, L Boccia, A De Rosa, R Di Palo, G Campanile, L Zicarelli. 2004. Chemical activation of buffalo (Bubalus bubalis) oocytes by different methods: effects of aging on post-partenogenetic development. Theriogenology 62, 1627-1637.

Gatica C. 2004. Estudio de dos protocolos de activación y condiciones de cultivo sobre el desarrollo de embriones partenogenéticos bovinos. Memoria de título. Escuela de Medicina Veterinaria, Universidad Austral de Chile, Valdivia, Chile.

Gonzáles PA. 2007. Desarrollo de embriones bovinos partenogenéticos y fecundados in vitro cultivados in vitro e in vivo en oviducto de coneja. Memoria de título. Escuela de Medicina Veterinaria, Universidad Austral de Chile, Valdivia, Chile.

Hochi S, K Ito, M Hirabayashi, M Ueda, K Kimura, A Hanada. 1998. Effect of nuclear stages during IVM on the survival of vitrifiedwarmed bovine oocytes. Theriogenology 49, 787-796.

Isachenko V, C Soler, E Isachenko, F Pérez-Sánchez, V Grishchenko. 1998. Vitrification of inmature porcine oocytes: effects of lipids droplets, temperature, citoskeleton, and addition and removal of cryoprotectant. Cryobiology 36, 250-253.

Martínez M, R Gatica, JE Correa, W Eyestone. 2007. Gestaciones producidas con embriones bovinos clonados por transferencia nuclear. Arch Med Vet 39, 59-62.

Martino A, N Songsasen, SP Leibo. 1996. Development into blastocysts of bovine oocytes cryopreserved by ultra-rapid cooling. Biol Reprod 54, 1059-1069.

Massip A, P Mermillod, A Dinnyes. 1995. Morphology and biochemistry of in-vitro produced bovine embryos: Implications for their cryopreservation. Hum Reprod 10, 3004-3011.

Rall WF, GM Fahy. 1985. Ice-free cryopreservation of mouse embryos at -196 degrees $C$ by vitrification. Nature $313,573-575$.

Ruiz JA, JE Correa, G Ayuque, L Landeo, M Yaranga, A Zacarías. 2007. Producción in vitro de embriones partenogenéticos de alpaca y llama. Resúmenes del I Congreso Nacional de Reproducción y Mejoramiento Genético de Camélidos Sudamericanos y I Simposium Internacional de Biotecnología Aplicada en Camélidos Sudamericanos. Huancavelica, Perú, Pp 145-149.

Ruiz JA. 2008. Avances en biotecnología reproductiva aplicada en la hembra de los camélidos sudamericanos. En: Quispe E (ed). Actualidades sobre adaptación, producción, reproducción y mejora genética en camélidos. $1^{\mathrm{a}}$ ed. Universidad Nacional de Huancavelica, Perú, Pp 49-82.

Saunders KM, JE Parks. 1999. Effects of cryopreservation procedures on the citology and fertilization rate of in vitro-matured bovine oocytes. Biol Reprod 61, 178-187.

Silva ME, MA Berland. 2004. Vitrificación de blastocitos bovinos producidos in vitro con el método Open Pulled Straw (OPS): Primer reporte. Arch Med Vet 36, 59-62.

Takahashi Y, NL First. 1992. In vitro development of bovine one-cell embryos: influence of glucosa, lactate, pyruvate, amino acids and vitamins. Theriogenology 37, 963-978.

Tanaka H. 1997. Rol de la activación del ovocito en la fecundación y el transplante de núcleos. AGCI e Instituto de Reproducción Animal. Universidad Austral de Chile, Valdivia, Chile.

Varga E, R Pataki, Z Lorincz, J Koltai, A Bali Papp. 2008. Parthenogenetic development of in vitro matured porcine oocytes treated with chemicals agents. Anim Reprod Sci 105, 226-233.

Williams C. 2002. Signalling mechanism of mammalian oocyte activation. Human Reprod Update 8, 313-321. 